\title{
Research on Paraffin Deposition Law and Paraffin Removal-Prevention Technology of Rodless Lifting Wellbore
}

\author{
Yuan Wen*, Jiang Wei, Wang Yang \\ Daqing Oilfield Production Engineering Research Institute, Daqing, China
}

Email address:

cyyyuanwen@petrochina.com.cn (Yuan Wen)

${ }^{*}$ Corresponding author

To cite this article:

Yuan Wen, Jiang Wei, Wang Yang. Research on Paraffin Deposition Law and Paraffin Removal-Prevention Technology of Rodless Lifting Wellbore. Petroleum Science and Engineering. Vol. 3, No. 1, 2019, pp. 29-33. doi: 10.11648/j.pse.20190301.15

Received: April 7, 2019; Accepted: May 23, 2019; Published: June 13, 2019

\begin{abstract}
The rodless lifting technology can overcome the shortcomings of rod and tube wear, high energy consumption and heavy maintenance workload in the lifting process of rod pumps, which is gradually being developed and adopted by major oil fields around the world. However, the paraffin deposition is coming more and more serious duo to non-sucker rod disturbance in wellbore and the relatively slow flow rate of well fluid, the production and maintenance cost are increasing, the pump inspection cycle is shortened, which creates a bottleneck for the further popularization of rodless lifting technology. Therefore, the research and development of rodless lifting oil well paraffin removal-prevention technology is great significance to the application and promotion of rodless lifting technology. Based on the study of paraffin deposition in oil wells, the effects of paraffin content, fluid flow rate, gelatin, wall temperature and other factors on paraffin deposition in crude oil are analyzed, to discuss the internal relationship. By the laboratory tests, the mathematical model of paraffin deposition in rodless lifting wellbore is obtained. Field tests were carried out to verify the paraffin control effect of the developed solid paraffin inhibitor matched with the paraffin deposition law and mathematical model of rodless lifting wellbore. Field tests show that the period of heat-free washing of rodless lifting wells is more than half a year after the application of solid paraffin inhibitors, and the effect of paraffin control is obvious. The research and development of paraffin removal and prevention technology is of great significance to the popularization and application of rodless lifting technology. The research and development of paraffin removal-prevention technology is important for the popularization and application of rodless lifting technology.
\end{abstract}

Keywords: Rodless Lifting, Deposition, Paraffin, Inhibitor, Test

\section{Introduction}

At present, pumping units and screw pumps are the main methods of mechanical production in major oilfields in China. The technology and matching technology are mature, however, in the application of some low-yield wells, directional wells and polymer flooding wells, problems such as serious rod and tube wear, high lifting energy consumption and heavy maintenance workload are exposed. For this reason, the research on rod-less lifting technology is carried out [1-4]. After years of tackling key problems, the main technology has been basically stereotyped and matched, and good application results have been achieved. However, during the application of sucker rod-less lifting technology, the serious paraffin deposition problem caused by the non-sucker rod disturbance in wellbore and the relatively slow flow rate of well fluid [5-7], which leads to the increase of production and maintenance costs, the shortening of pump inspection cycle. Those greatly restricts the popularization and application of sucker rod-less lifting technology.

Among the main paraffin removal-prevention technologies, the cost of paraffin removal by electric heating of hollow rod is high, the conventional well washing, high pressure hot washing and mechanical paraffin scraping are common methods. However, there are some problems such as the influence of reservoir pressure on production, to damage of cable and labor intensity, and mature plug removal technology is shorting. Statistical data shows that the proportion of pump 
checking caused by paraffin blockage in rodless hoist production wells exceeds $20 \%$, and the average pump checking cycle is less than one year, which is becoming a bottleneck restricting the popularization and application of this technology. It is urgent that carry out relevant technical researches [8-10]. Solid paraffin inhibitor has the advantages of low cost, maintenance-free and long effective time. It is an important part of prolonging the pump cycle of rodless lifting wells and reducing maintenance costs.

\section{Paraffin Deposition Law}

The precondition of applying solid paraffin inhibitor understand the paraffin deposit regularity of crude oil and the cause of paraffin deposit. paraffin deposition in oil wellbore is mainly explained by molecular diffusion theory. paraffin crystallization in crude oil precipitates and deposits on the inner wall of pipeline with gum, asphaltene, some crude oil and other impurities, which reduces pipe diameter, increasing friction of crude oil and decreases pipeline transportation capacity, and gradually forms paraffin deposition [11-13].

The research on paraffin deposition law mainly bases on experiments. The paraffin precipitation and deposition process are observed by paraffin deposition test device, and the relevant mathematical and theoretical models are established. Through a large number of experimental data, domestic and foreign researchers have found that paraffin content, temperature and oil flow rate are main factors affecting the paraffin deposition of crude oil, the components of crude oil in different blocks are different, such as the proportion of crude oil in the Middle East, Russia, Colombia and so on. By correctly understanding the paraffin deposition law of crude oil block that we can adopt the correct paraffin removal-prevention methods [14-15], carrying out paraffin removal and plug removal. This paper focuses on discussing the influence of the paraffin deposition law of oil wells on the matching, dosage and delivery cycle of solid paraffin inhibitors.

\subsection{Paraffin Content}

The effect of paraffin content in crude oil on paraffin deposition was studied under the conditions of oil temperature $50^{\circ} \mathrm{C}$, wall temperature $35^{\circ} \mathrm{C}$ and flow rate $1 \mathrm{t} / \mathrm{d}$. From the relationship curve between paraffin deposition rate and paraffin content in Figure 1, it can be seen that the paraffin deposition rate of crude oil increases first and then decreases with the increase of paraffin content.

The reason is that when the paraffin content in crude oil is at a low level, the paraffin deposition phenomenon increases with the increase of paraffin content; when the paraffin content increases to a certain value, the paraffin molecules present a three-dimensional network structure, which leads to the increase of the solidification point of crude oil and the deterioration of fluidity, increasing the shear effect of fluid on the inner wall of the tubing at the same flow rate, reducing the paraffin crystals attached to the inner wall of the tubing and paraffin deposition rate.

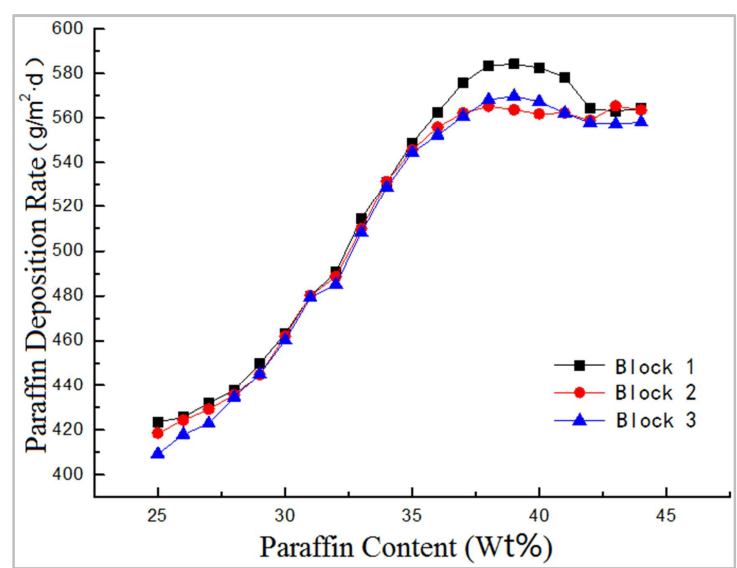

Figure 1. Curve between paraffin deposition rate and paraffin content.

\subsection{Tube Wall Temperature}

Figure 2 shows the relationship between the paraffin deposition rate and the tube wall temperature. showing the paraffin deposition rate of three blocks at different tube wall temperature. The paraffin deposition rate increases first and then decreases with increasing of tube wall temperature, reaching the maximum at $25^{\circ} \mathrm{C}$. When the tube wall temperature is low, the crude oil loses a lot power, dissipating heat quickly, to cause high viscosity. Paraffin molecules are easy to move to the tube wall, resulting in the deposition of paraffin molecules. Even if there is a large temperature difference between the crude oil and the tube wall, paraffin molecules will be carried away by the shear force of crude oil with high viscosity, resulting in the slow increase of paraffin deposition rate in the low temperature region. When the tube wall temperature rises to $25^{\circ} \mathrm{C}$, the crude oil viscosity decreases obviously. The paraffin molecule deposited near the tube wall, the paraffin deposition ability is better than the shear force of crude oil, resulting in a rapid increase of paraffin deposition rate at $25^{\circ} \mathrm{C}$. When the wall temperature exceeds $25^{\circ} \mathrm{C}$, the paraffin molecules precipitation performance become worse inside the crude oil. The paraffin molecules deposited near the wall decrease rapidly, with the temperature rising, the more paraffin molecules deposition is difficult. With the increase of temperature, the paraffin deposition rate decreases.

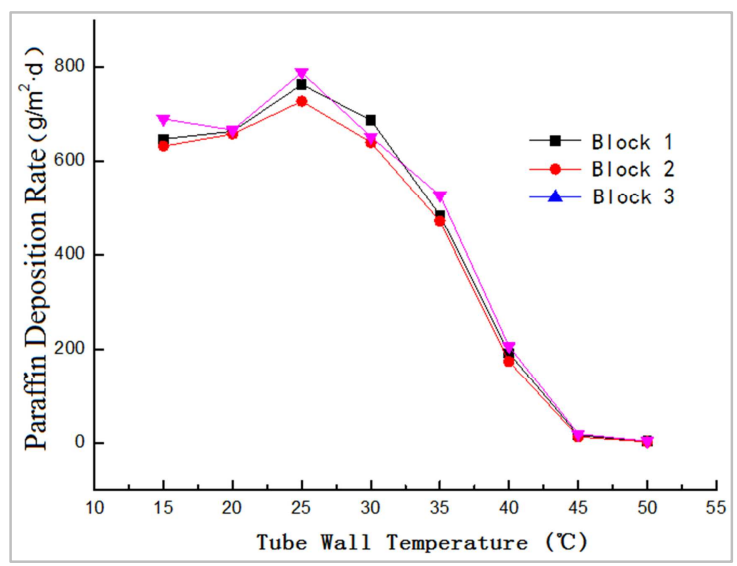

Figure 2. Curve between paraffin deposition rate and tube wall temperature. 


\subsection{Crude Oil Flow Rate}

Under the conditions of oil temperature of $50^{\circ} \mathrm{C}$ and tube wall temperature of $35^{\circ} \mathrm{C}$, the effect of crude oil flow rate on paraffin deposition was studied in laboratory. From Figure 3, it can be seen that the paraffin deposition rate of crude oil increases first and then decreases with the increase of flow rate. The flow rate increases resulting in the increase of crude oil flow speed. In the low flow rate region, the increase of flow rate results in the increase of heat dissipation of crude oil, the decrease of crude oil temperature, the increase of viscosity and the proximity of paraffin molecules. The shear force of crude oil in the low flow rate region is not enough to overcome the paraffin content. As the flow rate increases further, the flow rate and the effect of shear force on paraffin increasing further, the affinity between paraffin molecules become worse which is not easy to deposit, so the paraffin deposition rate decreases with the increase of flow rate.

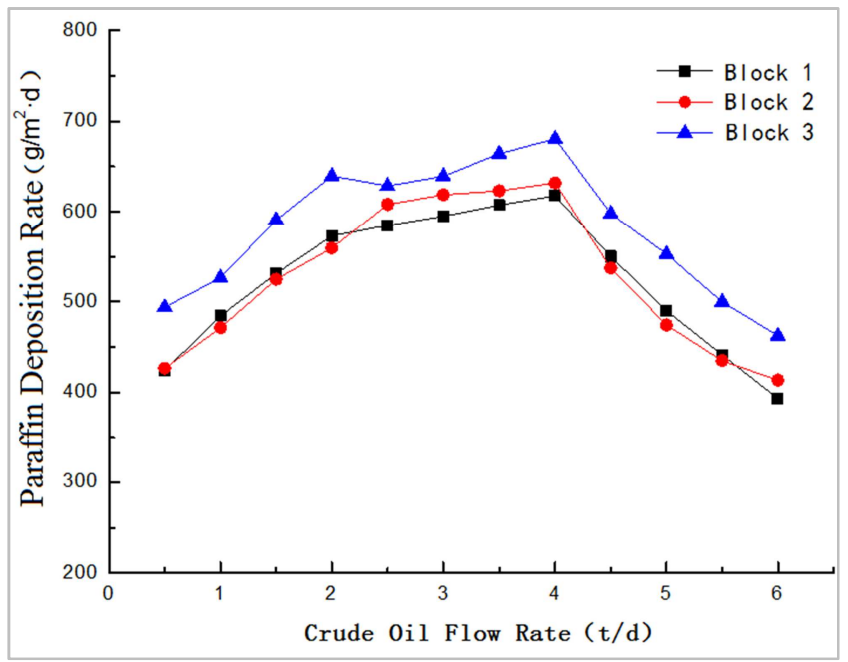

Figure 3. Curve between paraffin deposition rate and crude oil flow rate.

\subsection{Colloid and Asphalting}

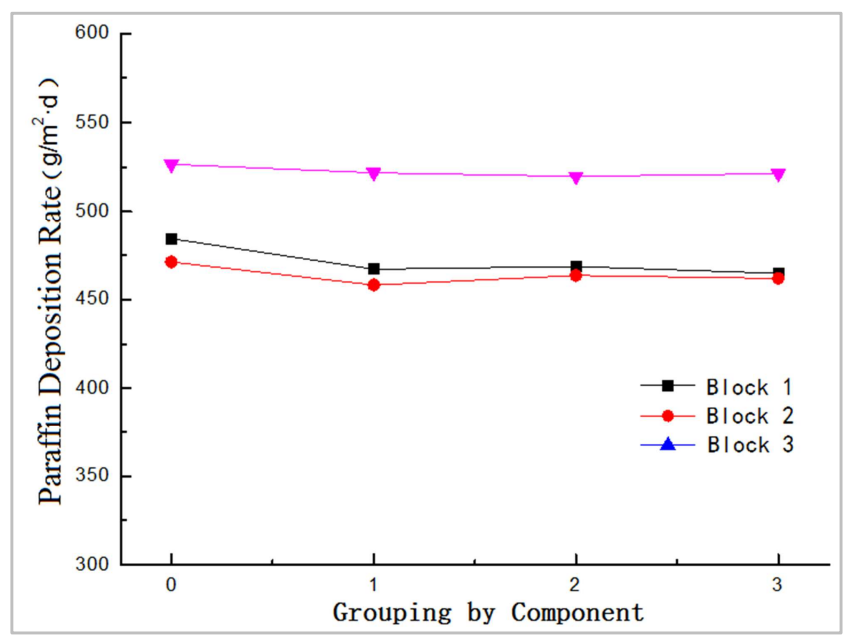

Figure 4. Curve between paraffin deposition rate and colloid and asphaltene.

Under the conditions of oil temperature $50^{\circ} \mathrm{C}$, wall temperature $35^{\circ} \mathrm{C}$ and flow rate $1 \mathrm{t} / \mathrm{d}$, we measured the crude oils with different components in laboratory. From the relationship between paraffin deposition rate with colloid and asphaltene content in Figure 4, it can be seen that colloid and asphaltene in crude oil has no obvious relationship with paraffin deposition rate. The specific performance is that the paraffin deposition rate of crude oil remains basically unchanged, with increasing of colloid and asphaltene,

\section{Paraffin Deposition Model}

The paraffin deposition law shows that paraffin content, tube wall temperature and flow rate are the main factors affecting paraffin deposition rate. According to the paraffin deposition law of crude oil in different paraffin content, tube wall temperature and flow rate, the mathematical model of paraffin deposition law of oil wells is established, such as Table 1, Table 2, Table 3. Different models match different paraffin removal and control measures to ensure that the oil well is in an ideal working state.

Table 1. Mathematical model of paraffin deposition rate and paraffin content.

\begin{tabular}{ll}
\hline Block & $\begin{array}{l}\text { Paraffin Deposition Law (V: Paraffin deposition rate, } \\
\left.\mathbf{g} / \mathbf{m}^{2} \bullet \mathbf{d} ; \mathbf{X} \text { : Paraffin content, Wt } \% \times \mathbf{1 0 0}\right)\end{array}$ \\
\hline Block1 & $\mathrm{V}=-0.567 \mathrm{x}^{2}+48.70 \mathrm{x}-468.0$ \\
Block2 & $\mathrm{V}=-0.512 \mathrm{x}^{2}+44.41 \mathrm{x}-394.1$ \\
Block3 & $\mathrm{V}=-0.585 \mathrm{x}^{2}+49.67 \mathrm{x}-488.2$ \\
\hline
\end{tabular}

Table 2. Mathematical model of paraffin deposition rate and tube wall temperature.

\begin{tabular}{ll}
\hline Block & $\begin{array}{l}\text { Paraffin Deposition Law (V: Paraffin deposition rate, } \\
\left.\mathbf{g} / \mathbf{m}^{2} \bullet \mathbf{d} ; \mathbf{X}: \text { Tube wall temperature, }{ }^{\circ} \mathbf{C}\right)\end{array}$ \\
\hline Block1 & $\mathrm{V}=0.067 \mathrm{x}^{3}-7.413 \mathrm{x}^{2}+229.3 \mathrm{x}-1398$. \\
Block2 & $\mathrm{V}=0.064 \mathrm{x}^{3}-7.060 \mathrm{x}^{2}+216.2 \mathrm{x}-1281$. \\
Block3 & $\mathrm{V}=0.058 \mathrm{x}^{3}-6.486 \mathrm{x}^{2}+199.5 \mathrm{x}-1089$ \\
\hline
\end{tabular}

Table 3. Mathematical model of paraffin deposition rate and flow rate.

\begin{tabular}{ll}
\hline Block & $\begin{array}{l}\text { Paraffin Deposition Law (V: Paraffin deposition rate, } \\
\left.\mathbf{g} / \mathbf{m}^{2} \cdot \mathbf{d} ; \mathbf{X} \text { : Flow rate, } \mathbf{t} / \mathbf{d}\right)\end{array}$ \\
\hline Block1 & $\mathrm{V}=-26.92 \mathrm{x}^{2}+167.8 \mathrm{x}+343.3$ \\
Block2 & $\mathrm{V}=-28.21 \mathrm{x}^{2}+177.3 \mathrm{x}+332.5$ \\
Block3 & $\mathrm{V}=-25.18 \mathrm{x}^{2}+157.5 \mathrm{x}+410.4$ \\
\hline
\end{tabular}

\section{Solid Paraffin Inhibitor}

\subsection{Theoretical Models}

The application of solid paraffin inhibitors is based on the law of paraffin deposition in oil wells and the mathematical model of paraffin deposition. Different wells condition should correspond to different solid paraffin inhibitors. For this reason, when studying the theoretical models of solid paraffin inhibitor, assuming that wells condition and the structure of the paraffin blocks are the same, the dynamic simulation test of the dissolution rate is carried out by using the similar principle and the dynamic simulation device to study the influence of the dissolution rate on the solid paraffin inhibitor.

Assuming that the geometry of the assume model is similar to prototype model, the Reynolds numbers of the two models 
are equal, then:

$$
\begin{aligned}
& \frac{D U}{v}=\frac{d u}{v} . \\
& U=\frac{Q}{\frac{\pi}{4} D^{2}} . \\
& u=\frac{q}{\frac{\pi}{4} d^{2}} . \\
& d=D \frac{q}{Q} .
\end{aligned}
$$

Formula: D- Oil well paraffin block inner diameter, m; U- Oil well paraffin block inner well fluid flow rate, $\mathrm{m} / \mathrm{s}$; d- Test paraffin block inner diameter, $\mathrm{m}$; $\mathrm{u}$ - Test paraffin block liquid flow rate, $\mathrm{m} / \mathrm{s}$; $\mathrm{v}$ - Well fluid motion viscosity, $\mathrm{cm} 2 / \mathrm{s}$;

Q- Oil well fluid production, $\mathrm{m} 3 / \mathrm{s}$; q-High pressure pump exclusion flow rate, $\mathrm{m} 3 / \mathrm{s}$. Principle of Geometric Similarity:

$$
l=L\left(\frac{d}{D}\right)
$$

Formula: L- Total length of paraffin block for oil well, m; 1- Total length of paraffin block for test, $\mathrm{m}$.

\subsection{Paraffin Inhibitors}

Relevant literature shows that the depth of the solid paraffin inhibitor in the tubing directly affects its working efficiency. When the depth is too deep, the temperature in the tubing is higher to lead the dissolution rate of the reagent becoming faster. Although the paraffin deposition in oil wells can be inhibited, the service life of the reagent becomes shorter, the power is wasted, the maintenance cost is increased by adding reagents frequently. When the depth is too shallow, the inner temperature of the tubing is lower, the dissolution rate of the reagent becomes slower, which is lower than the lowest use standard, the purpose of paraffin removal-prevention cannot be achieved. Therefore, the temperature in the tubing should be guaranteed to ensure that the solvent is carried out at the expected rate, so that the paraffin molecules in the crude oil can reduce affinity and prevent their coalescence during the cooling process of moving to the wellhead.

The depth and dosage of solid paraffin inhibitor depend on wellbore production. Assuming the use period is half a year, the dosage of paraffin inhibitor:

$$
\mathrm{M}=15 * \mathrm{q} *(1-\mathrm{fw}) * \mathrm{~d}
$$

Formula: M- solid paraffin block dosage, g; q- Measure liquid yield, $\mathrm{t} / \mathrm{d}$;

fw- Water content, \%;

d- Validity period.

\subsection{Field Tests}

In order to prove the application effect of solid paraffin inhibitors, field tests were carried out in a rodless lifting test area of one oil field. Five oil wells in the test area were injected with solid paraffin inhibitors. In order to investigate the application effect of solid paraffin inhibitor, samples analysis was carried out, the paraffin prevention rate of oil samples before and after using paraffin inhibitor was determined. Five wellhead oil samples without solid paraffin inhibitor and with solid paraffin inhibitor were tested. The results are shown in Table 4 and Table 5. It can be seen that the amount of paraffin deposited in oil wells decreases significantly after using solid paraffin inhibitors. The application of the technology also saves a lot of manpower and

\begin{tabular}{|c|c|c|c|c|c|c|}
\hline \multirow[b]{2}{*}{ No. } & \multicolumn{3}{|l|}{ Measure Before } & \multicolumn{3}{|l|}{ Measure After } \\
\hline & $\begin{array}{l}\text { Liquid Production } \\
\text { t/d }\end{array}$ & Oil Producion t/d & $\begin{array}{l}\text { Percentage of } \\
\text { Moisture \% }\end{array}$ & $\begin{array}{l}\text { Liquid Production } \\
\text { t/d }\end{array}$ & Oil Producion t/d & $\begin{array}{l}\text { Percentage of } \\
\text { Moisture \% }\end{array}$ \\
\hline 1 & 1.5 & 0.4 & 70.3 & 4.3 & 1.0 & 76.2 \\
\hline 2 & 3 & 2.3 & 22.6 & 2.4 & 1.5 & 35.6 \\
\hline 3 & 2.6 & 0.8 & 69.3 & 3.0 & 0.8 & 74 \\
\hline 4 & 2 & 0.7 & 66.2 & 2.1 & 0.7 & 66.2 \\
\hline 5 & 1.6 & 1.3 & 21.6 & 1.4 & 1.1 & 21.6 \\
\hline
\end{tabular}
material resources, facilitates production management, which has one great social benefits.

Table 4. Paraffin control rate after using solid paraffin inhibitor.

\begin{tabular}{ll}
\hline Samples & Paraffin Control Rate \% \\
\hline 1 & 64.6 \\
2 & 63.2 \\
3 & 65.8 \\
4 & 66.9 \\
5 & 60.3 \\
\hline
\end{tabular}

Table 5. Oil wells statistical table of the situation of solid solid paraffin inhibitor.

\section{Conclusion}

Paraffin deposition in crude oil is closely related to paraffin content, tube wall temperature and flow rate, but not to colloid and asphaltene. Through simulation experiments, the following paraffin deposition laws are obtained:
1. When the paraffin content in crude oil is low, the paraffin deposition rate increases rapidly with the increase of paraffin content, while when the paraffin content is higher than $35 \%$, the paraffin deposition rate of crude oil slows down obviously.

2. With the decrease of wall temperature, the paraffin 
deposition rate increases first and then slows down, and reaches the highest value at about $25^{\circ} \mathrm{C}$.

3 . With the increase of flow rate, the paraffin deposition rate increases first and then decreases, and reaches the highest value around $4 \mathrm{t} / \mathrm{d}$.

4. The paraffin deposition rate decreases to a certain extent with the decrease of asphaltene and gum content, but the correlation with asphaltene and gum content is not significant, indicating that the content of asphaltene and gum in crude oil is not the main factor causing paraffin deposition in wellbore.

5. According to the paraffin deposition law of crude oil in each block under different paraffin content, pipe wall temperature and flow rate, a mathematical model of paraffin deposition law was established, ensuring that oil wells are in good production condition.

The application of solid paraffin inhibitor in oil wells can achieve a period of no-heat washing for more than half a year. The paraffin control rate of 5 wellhead oil samples before and after using solid paraffin inhibitor was determined. The results showed that the paraffin deposition rate of oil wells decreased significantly after using solid paraffin inhibitor. In the next research, the emphasis will be on increasing the paraffin control cycle of solid paraffin inhibitors. Under the same amount of paraffin inhibitors, the paraffin control cycle of oil wells will be delayed and the operation cost will be reduced.

The paraffin deposition law and mathematical model of rodless lifting wellbore can provide effective theoretical guidance for the application of solid paraffin inhibitors, improving the paraffin prevention rate of solid paraffin inhibitors, prolong the pump inspection cycle of rodless lifting wells, reducing maintenance costs. It is one great significance for popularizing and applying of rodless lifting technology.

\section{References}

[1] Meng Lingkai. Research on the Prediction Method of Eccentric Wear Life between Rod and Tube for Flooding Pumping Well [D]. Qinhuangdao, Yanshan University, 2016.

[2] Zhu Zhengjun. Establish Comprehensive Techniques for Controlling Friction between Rod and Tube Pumped Wells in HB Ttest Areas [D]. Daqing, Northeast Petroleum University, 2016.
[3] Li Hongkang. Research and Application of Comprehensive Anti-Partial Wear Strategy in H301 Block [D]. Daqing, Northeast Petroleum University, 2018.

[4] Wang Zhengyu, Wu Weidong. Statistical Analysis and Study on the Pump Inspection Period for Mechanical Wells in Daqing Oilfield [J]. Oil Production Engineering, 2018, 1, 81-86.

[5] Bai Zhonggang. Application of Comprehensive Paraffin Removal and Prevention Technology in Oilfield [J], Petrochemical Industry Technology, 2017, 10, 262.

[6] Luoyi. Paraffin Removal and Control in Late Stage of Oilfield Development [J]. Petrochemical Industry Technology, 2017, $12,25$.

[7] Wang Chunxia, Tian Xiaohong, Wang shuhai. Analysis and Understanding of Paraffin Deposition Control Effect in Oil Wells [J]. Chemical Enterprise Management, 2018, 3, 224.

[8] Zhang Wanqing. Study on Paraffining Law and Solid Paraffin Inhibitor of Rodless Life Wellbore [D]. Daqing, Northeast Petroleum University, 2018.

[9] Zhengshuai. Design of Rodless Lifting Paraffin Control Inverter Heating System [D]. Harbin, Harbin University of Science and Technology, 2015.

[10] Liu Yuguo, Yao Anlin, Shi Hanchen. Discussion on the Causes of Oil Well Deposition and Paraffin Prevention Measures [J]. Modern Chemical Research, 2018, 4, 5-6.

[11] Liu Jingchen. Study on Paraffining Characteristics and Paraffin Removal Technology of Oil Well [D]. Chengdu, Southwest Petroleum University, 2017.

[12] Wang Bojun. Research on Downhole Solid Scale and Paraffin Control Technology [D]. Daqing, Daqing Institute of Petroleum, 2006.

[13] Pan Huafeng. Application of Paraffin Control Energy-Saving Technology in Pumping Well $[\mathrm{J}]$. Energy Conservation in Petroleum \& Petrochemical Industry, 2013, 11, 23-26.

[14] Zhang Yunjie. Evaluation of Application Effect of Solid Paraffin Preventer in Low Production Wells [J]. Energy Conservation in Petroleum \& Petrochemical Industry, 2012, 6, 35-36.

[15] Lv Wenkai, Zhou Guijun, Lv Liping. Application Effect Analysis of Downhole Solid Paraffin Control and Pour Point Depression Technology [J]. Energy Conservation in Petroleum \& Petrochemical Industry, 2011, 9, 30-31. 